\title{
Enhanced lightweight NVH solution based on vibro-acoustic metamaterials
}

\author{
C. Claeys, E. Deckers, B. Pluymers, W. Desmet \\ KU Leuven, Noise and Vibration Research Group, member of Flanders Make.
}

\begin{abstract}
The NVH performance of conventional panels and structures is mainly driven by their mass. Vibro-acoustic metamaterials with stopband behaviour are looked upon as a possible solution for combining NVH and lightweight requirements for engineering structures. This article discusses a metamaterial concept on based embedded resonant structures that exhibit vibro-acoustic stopband behaviour. This potential is shown through the design and validation of a demonstrator for increased acoustic insertion loss and a demonstrator for improved vibration attenuation. These metamaterials can be achieved through a variety of materials, designs or production processes, depending on the application, and can offer technological benefits such as possible integration in structural parts, use in harsh environments and ease of design of the beneficial frequency ranges, paving the way for a new class of light and compact NVH solutions with ample applications in automotive for low and mid frequency NVH mitigation.
\end{abstract}

\section{Introduction}

The Noise and Vibration Harshness (NVH) behaviour of products is gaining importance due to increasing customer expectations and more stringent regulations. Ecological trends, however, increase the importance of lightweight design and reduce the applicability of classic (heavy) solutions. In view of this challenging and often conflicting task of merging $\mathrm{NVH}$ and lightweight requirements novel solutions are required.

Vibro-acoustic metamaterials are candidates for superior lightweight NVH insulation, be it at least in targeted frequency ranges, referred to as stopbands. These stopbands result from resonant cells arranged on a subwavelength scale [1][2][3]. Previously, the authors explained the working principles, listed driving parameters and introduced a metamaterial concept based on embedding resonant structures [4][5]. This article shows the potential for acoustic insulation and vibration reduction in a low frequency zone.

This article is structured as followed. Section 2 discusses the rationale behind the proposed metamaterial concept and the numerical prediction of stopband behaviour. Section 3 shows the potential of this concept for acoustic reduction, while section 4 discusses a demonstrator for vibration reduction. The article ends with the conclusions.

\section{Metamaterials by inclusion of resonant structures}

To obtain metamaterials with stopband behaviour two conditions need to be met; (i) resonant 
cells have to be added to a host structure on a scale smaller than the structural wavelengths to be influenced [1] and (ii) the net sum of the forces on the hosting structure contributed by a resonant system should be non-zero [6].

The kinds of resonant systems which are eligible heavily depend on the structure to which the resonant systems are added. As a first example periodic lightweight structures, such as honeycomb or rectangular core sandwich panels, are considered. The internal cavities of these periodic core sandwich panels allow inclusion of the resonant systems while the combination of excellent mechanical properties and low mass can be preserved.

A huge number of designs can be proposed for the resonant structures, leaving room for optimisation towards e.g. minimal weight or maximal attenuation. For this article a straightforward design is chosen that resembles a mass-spring system; two thin legs are used to connect a heavy mass to a host structure (Figure 1). The connection legs will determine the stiffness while the thick part of the resonator will determine the mass of this resonant structure.
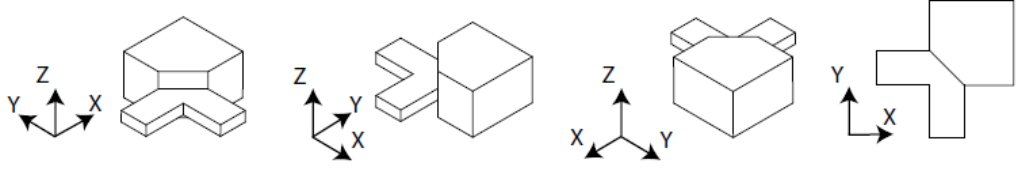

Figure 1: Resonant structure used in the acoustic demonstrator to introduce stopband behaviour.

To assess whether the addition of these resonant structures to the cavities of a periodic sandwich core will introduce a stopband, the wave propagation needs to be investigated. From literature it is known that wave propagation through infinite periodic structures can be investigated through unit cell modelling [7][8]. Based on an undamped Finite Element (FE) model of the unit cell and the application of periodicity boundary conditions, dispersion curves for freely propagating waves in an infinite periodic structure can be derived. Frequency zones for which no solutions are found, correspond to frequency zones without free wave propagation and thus a stopband region. Thus by building a FE model of the unit cell metamaterial, both the resonance frequency of the resonant structure as the dispersion curves can be calculated.

\section{Acoustic demonstrator}

To prove the potential of the introduced metamaterial concept to reduce acoustic transmission, acoustic enclosures with one open side are designed. These can be placed over a small speaker such that the acoustic insertion loss can be determined by comparing sound radiation with and without enclosure.

Figure 2 shows the demonstrator, made through Selective Laser Sintering. The inner volume is $100 \times 100 \times 100 \mathrm{~mm}$, the ribs are hollow and each side consists of $8 \times 8$ unit cells.

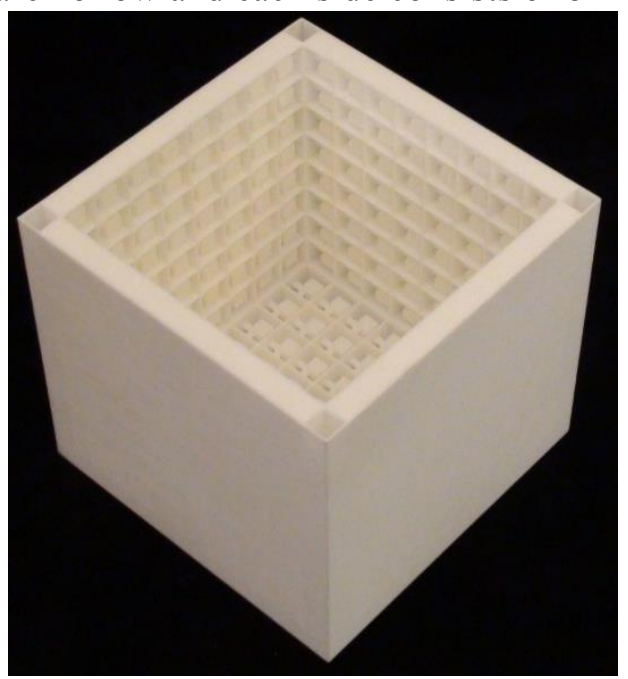

Figure 2: Picture of the acoustic demonstrator.

Figure 3 compares the measured insertion loss of the metamaterial demonstrator to an enclosure 
with the same weight and produced in the same way but with flat sides of $3.5 \mathrm{~mm}$ thickness. Between 700 and $1000 \mathrm{~Hz}$ the demonstrator enclosure clearly outperforms the regular enclosure. To see and hear this effect, the interested reader is referred to following link: http://youtu.be/tOch GsGaXg. The frequency zone of improved acoustic behaviour is a bit wider than predicted by the stopband simulation $(886-999 \mathrm{~Hz})$, but this corresponds well with previous simulations of the authors [4].

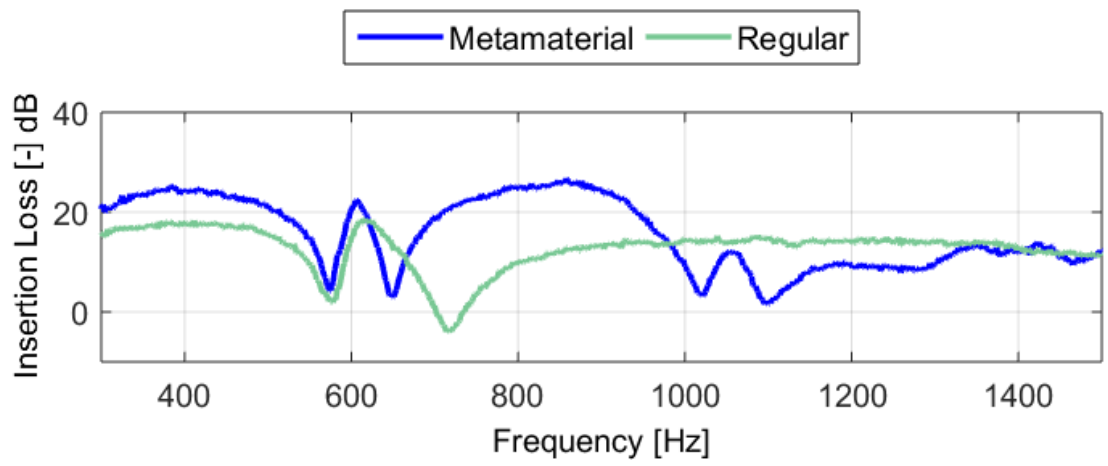

Figure 3: Comparison between the measured insertion loss for the metamaterial demonstrator and an equivalent mass enclosure with flat side panels and the same weight.

\section{Vibration reduction demonstrator}

To prove the potential of metamaterials for vibration reduction along a transmission path, resonant structures are added to a PVC duct of outer dimension $100 \times 100 \times 1000 \mathrm{~mm}$ and $2 \mathrm{~mm}$ thickness. The resonant structures are made through laser cutting a Plexiglas plate and are designed as cantilever beams with an end point mass, and a supporting foot to glue the structures to the duct. Figure 4 shows the resonant structures and a section of the duct. Depending on the mass at the end of the structure, the resonance frequency of the resonant structure can be changed, for these design the resonances are predicted at $512 \mathrm{~Hz}$ and $577 \mathrm{~Hz}$ for type A and type B respectively. More information on this design and the measurements can be found in references [9][10].

The demonstrators have as goal to show the effect of metamaterials on wave propagation and allow investigation of the possibility to combine stopbands. Therefore five different configurations will be considered as listed in Table 1. The mixed configuration combines both resonant structure types in a checkered pattern, as illustrated in Figure 4, while the sequential configuration has only type A resonant structures on the first half of the duct and only type B on the second half.

Table 1: Metamaterial characteristics of the different configurations: amount of added resonant structures of each type, mass addition relative to the bare duct and predicted stopband width.

\begin{tabular}{|l|l|l|l|l|}
\hline Configuration & \# Type A & \# Type B & Mass addition & Stopband width [Hz] \\
\hline original & 0 & 0 & $0 \%$ & $/$ \\
\hline A & 640 & 0 & $27.4 \%$ & $488-538$ \\
\hline B & 0 & 640 & $26 \%$ & $549-603$ \\
\hline sequential & 320 & 320 & $26.7 \%$ & $488-538$ \& 549-603 \\
\hline mixed & 320 & 320 & $26.7 \%$ & $494-513 \& 558-588$ \\
\hline
\end{tabular}




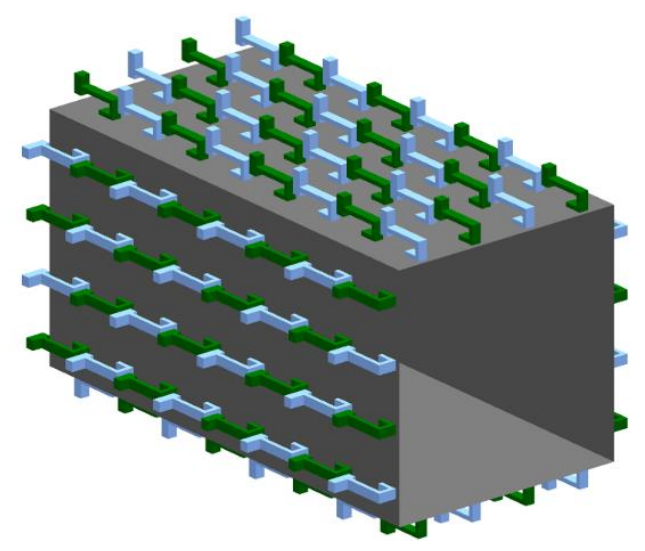

Figure 4: Visualisation of the resonant structure lay-out for the mixed configuration with green and blue resonant structures representing type A and type B respectively.

To measure the wave propagation along the duct, response measurements are made on different sections along the duct. A section is analysed by measuring three equidistant points on every face of a section between two circumferential rows of resonant structures, resulting in 12 measurements for each section. With those 12 points per section a RMS response is calculated. Since there are 40 circumferential rows along the duct, 41 sections can be defined; section 0 to section 40 .

Figure 5 compares the attenuation from section 4 to section 36 for the different configurations. The sequential configuration clearly combines the effect of both stopbands, but the overall width is slightly narrower than in the A or B configuration, which is due to less resonant structures of each type being present on the duct. The obtained results for the mixed and sequential configuration are similar, however, the mixed case shows a better overlap between the two stopbands regions.

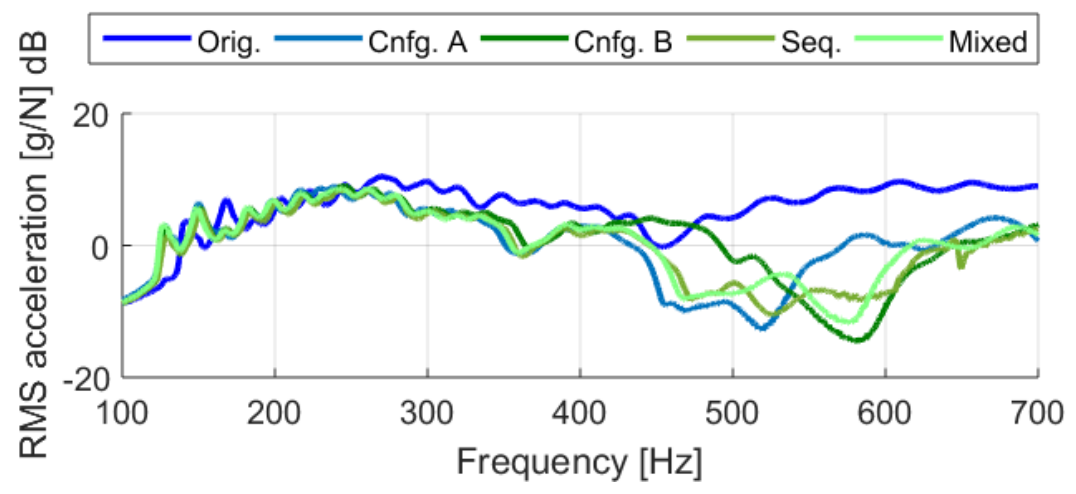

Figure 5: RMS acceleration for all configurations at section 36 for excitation in section 4

The difference between the sequential and mixed configuration, becomes apparent when exciting in the middle (section 20) and evaluating on the ends of the pipe (section 4 and section 36). Figure 6 shows that in the sequential case only the low frequent stopband is found going from section 20 to 4 and the high frequent stopband when going from section 20 to section 36 . In the mixed case, however, both stopbands are always present, although less pronounced. 


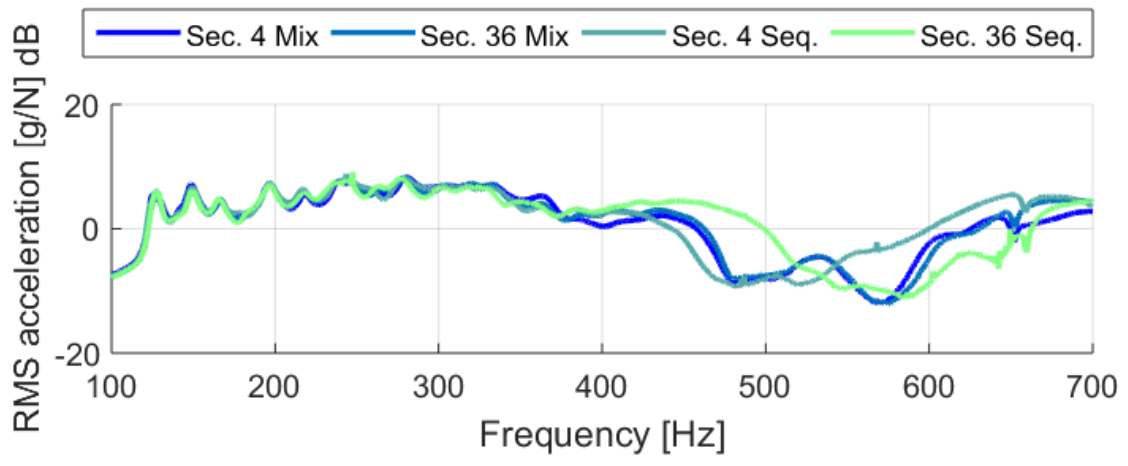

Figure 6: RMS acceleration for sequential (Seq.) and mixed (Mix) configurations at section 4 (Sec. 4) resp. 36 (Sec. 36) for excitation in section 20.

\section{Conclusions}

This paper introduces a novel method to create resonant metamaterial based NVH insulation: the addition of resonant structures to a host structure. Through the design of an acoustic demonstrator it is shown that this results in a frequency zone of increased acoustic insertion loss with respect to equivalent materials of the same weight. This potential can be seen and heard on the following link http://youtu.be/tOch GsGaXg. A vibrational demonstrator showed that different stopbands can be combined in a metamaterial to achieve wider stop bands. This can be done both by a mixed or sequential lay-out. The design of these metamaterials is aided by unit cell modelling: this tool allows a quick estimation of the location of the stopband frequencies and can be used to assess changes in resonant structure design. This research was partially funded by Flanders Make.

\section{Acknowledgments}

The authors would like to acknowledge N. Rocha de Melo Filho and L. Van Belle for their help with the measurements and simulations. Furthermore, the European Commission is acknowledged for their support through the ENLIGHT-project (http://www.project-enlight.eu/) and the KU Leuven Research Fund for their support through an IOF-Leverage project. Elke Deckers is a postdoctoral fellow of the Fund for Scientific Research Flanders (F.W.O.). The research of N. Rocha de Melo Filho is funded by a PhD Scholarship Sciences without Borders CNPq Brazil (201414/2014-7). The research of L. Van Belle is funded by a grant from the Research Foundation- Flanders (F.W.O.). This research was partially supported by Flanders Make.

\section{Authors}

Dr. Ir. C. Claeys: Claus Claeys is senior researcher in the KU Leuven Noise and Vibration Research Group, member of Flanders Make, supervising the metamaterial related research and managing metamaterial related research projects with industry.

Dr. Ir. E. Deckers: Elke Deckers is senior researcher in the KU Leuven Noise and Vibration Research Group, member of Flanders Make, supervising the research activities on lightweight design and vibro-acoustics.

Dr. Ir. B. Pluymers: Bert Pluymers is Senior Industrial Research Manager for the KU Leuven Noise and Vibration Research Group, member of Flanders Make, coordinating the tech transfer and valorization activities of the Research Group. 
Prof. Dr. Ir. W. Desmet: Wim Desmet is Full Professor at the KU Leuven Department of Mechanical Engineering, member of Flanders Make, where he heads the Noise \& Vibration Research Group. He also serves as Director of the Mechatronics and Design Department of Flanders Make, the strategic research centre for the manufacturing industry.

\section{References}

[1] J B. Pendry, D.J. Holden, A J. and Robbins, and W.J. Stewart, Magnetism from conductors and enhanced nonlinear phenomena, Microwave Theory and Techniques, IEEE Transactions on 47 (1999), no. 11, 2075-2084.

[2] F. Lemoult, N. Kaina, M. Fink, and G. Lerosey, Wave propagation control at the deep subwavelength scale in metamaterials, Nature Physics 9 (2012), 55-60.

[3] C. Goffaux, J. S'anchez-Dehesa, A.L. Yeyati, P. Lambin, A. Khelif, JO Vasseur, and B. DjafariRouhani, Evidence of fano-like interference phenomena in locally resonant materials, Physical Review Letters 88 (2002), no. 22, 225502.

[4] C. Claeys, K. Vergote, P. Sas, and W. Desmet, On the potential of tuned resonators to obtain low frequency vibrational stop bands in periodic panels, Journal of Sound and Vibration 332 (2013), no. 6, $1418-1436$.

[5] C. Claeys, E. Deckers, B. Pluymers, W. Desmet, "A lightweight vibro-acoustic metamaterial demonstrator: numerical and experimental investigation”, Mech. Syst. Signal Process., 2015, 853-880

[6] G. Wang, X. Wen, J. Wen, L. Shao, Y. Liu, "Two-dimensional locally resonant phononic crystals with binary structures", Physical Review Letters, 93(15), 2004, 154302

[7] L. Brillouin, Wave propagation in periodic structures, 2nd ed., McGraw-Hill Book Company, 1946.

[8] R.S. Langley, A note on the force boundary conditions for two-dimensional periodic structures with corner freedoms, Journal of Sound and Vibration 167 (1993), 377-381.

[9] N. Rocha de Melo Filho, C. Claeys, E. Deckers, B. Pluymers, W. Desmet, Dynamic metamaterials for structural stopband creation, ISNVH 2016, Graz, Austria

[10] C. Claeys, N. Rocha de Melo Filho, L. Van Belle, E. Deckers, B. Pluymers, W. Desmet, Design and validation of metamaterials for multiple structural stopbands in waveguides, Extreme Mechanics Letters (under review) 